\title{
Felicidad y Engagement en estudiantes-trabajadores de nivel superior en Lima
}

\author{
Engagement and Happiness levels in Lima's working college students
}

\author{
Katherine Abigail Davey Contreras ${ }^{1}$ \\ Universidad Privada de Ciencias Aplicadas
}

Recibido: $07-10-17$

Aceptado: $21-11-17$

\section{Resumen}

El presente trabajo de investigación tiene como finalidad estudiar si existe relación entre la felicidad y el engagement en estudiantes de educación superior que trabajan. Para ello, se realizó un estudio descriptivo correlacional de corte transversal. Se evaluó a un total de 338 estudiantes de centros de estudios superiores (universidades e institutos superiores). Los datos se obtuvieron utilizando la Escala de Felicidad de Lima, con una validez de criterio con la escala de afectos positivos de $\mathrm{r}=.48, \mathrm{p}<.01$ y confiabilidad de $\alpha=.916$ y el Cuestionario Engagement (UWES) con una validez de contenido V de Aiken $>0.80$ y confiabilidad de $\alpha>.77$, para la muestra de estudio. La Escala de Felicidad posee los siguientes factores: ausencia de sufrimiento profundo, realización personal, satisfacción con la vida, alegría de vivir, mientras que el cuestionario de engagement toma en cuenta los componentes vigor, dedicación y absorción. Hay relación entre felicidad y engagement, encontrándose en un nivel moderado bajo. La variable felicidad tuvo mayor correlación con los componentes dedicación y vigor del engagement. Los factores ausencia de sufrimiento profundo y satisfacción con la vida de la variable felicidad se correlacionan significativamente con los componentes dedicación y vigor del engagement.

Palabras clave: Psicología; Felicidad; Engagement

\section{Abstract}

This paper is meant to study if there is a relation between happiness and engagement in graduate students who work at the same time. To do this, a descriptive correlational study that features a cross-sectional design was carried out. 338 students from private institutions were evaluated (Universities and colleges).The data were obtained using the Happiness scale of Lima, with a criterion validity of the scale of positive affect of $r=.48, p<.01$ and reliability $\alpha=.916$, and the Engagement questionnaire (UWES), whith a V content validity Aiken $>$ 0.80 and reliability of $\alpha>.77$. The Happines scale has the following factors: absence of deep suffering, personal fulfillment, life satisfaction, 'joie de vivre', while the Engagement questionnaire takes into account the components of vigor, dedication and absorption. Statistically

1 Docente Universidad Privada de Ciencias Aplicadas, pchukdav@upc.edu.pe es

Nota de reconocimiento: La autora agradece los aportes académicos de María Atalaya, Alex Grajeda, Juan Kaneko, Carmen Meléndez, Mildred Paredes y Vladimir Terbullino.

(C) Los autores. Este artículo es publicado por la Revista de Investigación en Psicología de la Facultad de Psicología, Universidad Nacional Mayor de San Marcos. Este es un artículo de acceso abierto, distribuido bajo los términos de la licencia Creative Commons Atribucion - No Comercia_Compartir Igual 4.0 Internacional. (http://creativecommons.org/licenses/by-nc-sa/4.0/) que permite el uso no comercial, distribución y reproducción en cualquier medio, siempre que la obra original sea debidamente citada. 
significant correlations between happiness and Engagement were found in students working. The happiness variable had the highest correlation with the components of Engagement: dedication and then with vigor. The factors of profound suffering absence and life satisfaction that belongs to the happiness variable correlate significantly with the components of Engagement: dedication and vigor.

Keywords: Psychology; Happiness; Engagement

La Psicología, como disciplina, se ha orientado históricamente al estudio de la patología, y esto fue extensivo en las diferentes áreas que han ido surgiendo en esta ciencia, entre ellas la Psicología del trabajo y de las organizaciones. No obstante, en esta última década, la Psicología Positiva, sobre todo a partir de Seligman (2006) y Csikszentmihalyi (2008), ha empezado a cobrar mayor relevancia como prospecto de un nuevo paradigma en la Psicología, centrándose en los aspectos más adaptativos del ser humano, como la felicidad y el engagement.

La presente investigación se ubica dentro de esta corriente de la Psicología y plantea el estudio de la correlación de los conceptos de felicidad y engagement. Los objetivos son los siguientes: 1) determinar si existe relación entre felicidad y engagement en estudiantes que cursan el nivel superior y trabajan en Lima, 2) identificar el nivel de felicidad y engagement en esta población, y 3) determinar si existe relación entre los factores de la felicidad y los componentes de engagement en este mismo grupo.

Ambos constructos, felicidad y engagement se orientan al sentimiento de logro, por ello es relevante estudiar la relación entre ambos debido al interés de las organizaciones por tener trabajadores apasionados con sus labores, que disfruten de sus funciones y aporten en relación con una actitud positiva y una autopercepción optimista de sí mismos frente a sus vidas. Desde el ámbito de las universidades, debe ser de interés para las áreas concernientes al bienestar universitario reconocer que un porcentaje de sus estudiantes requiere trabajar para sostener sus gastos y/o ganar experiencia laboral. Por lo tanto, es valioso evaluar en qué nivel se encuentra la felicidad y engagement de los estudiantes que a la vez trabajan, ya que el nivel de demandas de responsabilidades es mayor y por lo tanto la percepción que tengan de sus recursos emocionales favorece en su desempeño. La postura de la presente investigación es Eudemónica (Padrós, 2002; Carr, 2007) y evidencia el estado mental positivo de los participantes. En este sentido, se considera que la felicidad y engagement se alcanzan por la realización del propio potencial.

Sobre el concepto de felicidad, se considera como la experiencia afectiva particular de una persona para sentir satisfacción en toda su plenitud ante la pertenencia de un bien que es altamente deseado. (Alarcón, 2009, p. 137). Ese bien anhelado puede ser una cosa, una persona, una situación, una vivencia, etc.; a su vez, los elementos que producen felicidad son de diversa índole (material, estético, ideológico, etc.). Por lo tanto, la cosa deseada no posee un valor intrínseco 
y un mismo bien no siempre genera la felicidad de todos los individuos. Para su evaluación, Alarcón (2006) elaboró una Escala de Felicidad de Lima con cuatro factores: ausencia de sufrimiento profundo, realización personal, satisfacción con la vida y alegría de vivir.

El primero de los factores, ausencia de sufrimiento profundo, consiste en sentir ausencia de depresión, con afecto positivo hacia uno mismo y hacia la vida. La segunda, satisfacción con la vida, manifiesta un sentimiento profundo de logro. El individuo se considera satisfecho con lo alcanzado en varios aspectos de su vida por lo que se acerca a una existencia ideal. El tercer factor, realización personal, expresa plenitud de felicidad permanente y no solo episodios esporádicos de la misma. Marca autosuficiencia, autarquía, equilibrio emocional, que condicionan a conseguir el estado de felicidad completa. Finalmente, alegría de vivir está orientada a la sensación de maravillarse con la vida y a ser positivo con lo que se vivencia.

En la presente investigación, se considera que felicidad difiere de conceptos como bienestar psicológico, aunque, en ciertas investigaciones (por ejemplo, Salas, 2010), se utilizan de manera intercambiable como términos generales que abarcan sentimientos y/o a actividades positivas (Seligman, 2006). Alarcón opta por utilizar el término "felicidad" debido a que explica por sí mismo el estado afectivo al cual se refiere y las características propias de la conducta feliz. El vocablo "felicidad" es semánticamente más complejo, posee valor explicativo y es más rico en contenido que el vocablo "bienestar subjetivo". Asimismo, Alarcón demuestra que la felicidad tiene su propia manera de operacionalizarse.

Respecto al engagement, es definido por Salanova, Schaufeli, GonzálesRomá y Bakker (2001) como un constructo motivacional positivo relacionado con el trabajo que está caracterizado por vigor, dedicación y absorción. Más que un estado específico y temporal, el engagement es un estado cognitivo-afectivo persistente en el tiempo, que no está focalizado sobre un objeto o conducta específica. Se operacionaliza con el puntaje obtenido al aplicar el Cuestionario engagement (UWES) de Salanova, Schaufeli, Llorens, Peiro y Grau (2000).

Con respecto a los componentes del engagement, se definen del siguiente modo, siguiendo a Salanova y Schaufeli (2009). Primero, vigor se refiere a altos niveles de energía y resistencia mental mientras se trabaja por el deseo de esforzarse en el trabajo que se está realizando, incluso cuando se presentan dificultades. Este es un componente conductual-enérgico. Segundo, la dedicación implica una alta implicación laboral, junto con la manifestación de un sentimiento de significación, entusiasmo, inspiración, orgullo y reto por el trabajo. Es un componente emocional. Tercero, la absorción es el estado en el que la persona está totalmente concentrada en su trabajo. El tiempo pasa rápidamente y presenta dificultades en el momento 
en que quiere desconectarse de lo que está haciendo, debido a las fuertes dosis de disfrute y concentración experimentadas. Este es un componente cognitivo.

Salanova, Grau, Llorens y Schaufeli (2001) empiezan a establecer el concepto engagement en contraposición con el burnout. Posteriormente, Salanova y Schaufeli (2009) plantean lo siguiente al respecto: "El burnout se caracteriza por una combinación de poca energía (agotamiento) y poca identificación (cinismo), mientras que el engagement en el trabajo se caracteriza por el patrón opuesto: una combinación de alta energía (vigor) y una elevada identificación (dedicación)" ( $p$. 109). Otros investigadores se han ocupado de enfocar sus estudios específicamente en el burnout (Manzano, 2002; Salas, 2010; Quintanilla, 2013; Glaría, Carmona, Pérez y Parra, 2015).

El engagement es un concepto psicológico novedoso, importante para el ámbito de los negocios y académicos, puesto que este estado psicológico acompaña a la conducta de mejora y energía personal, y se constituye como un elemento motivacional y social. Justamente por ello, se considera de relevancia relacionar este concepto con el de felicidad en estudiantes de educación superior que trabajan en Lima.

Con respecto al contexto en el que se inserta esta población, según la Organización Internacional del Trabajo - OIT (2015), la crisis económica de los años 2008-2009 ha generado un impacto negativo en la tasa de ocupación desde el segundo trimestre de 2013 en toda América Latina y el Caribe. Otra dificultad adicional es el empleo informal, en el que Perú, junto con Bolivia y Paraguay, presenta las mayores dimensiones, pues solo 3 jóvenes de cada 10 acceden a empleo formal, al margen de la protección social (OIT, 2011). Como consecuencia, Perú ocupa uno de los últimos lugares en América Latina en el ranking global de felicidad desde el 2013 (Costa, 2015). El contexto de necesidad económica es el que configura el hecho de que el 50\% aproximadamente decidan estudiar y trabajar a la vez (León y Sugimaro, 2013), pues ello les permite insertarse más rápido al mercado laboral y poder solventar sus estudios superiores. En este sentido, es significativo un estudio para esta población que trabaja y estudia que se incrementa cada vez más y que se enfrenta a una dinámica de vida que puede resultar agobiante si es que no se utilizan las estrategias correctas para afrontarla desde un punto de vista de la salud psicológica.

\section{Revisión bibliográfica sobre Felicidad y Engagement}

La única investigación en la cual se relaciona felicidad y engagement, como se propone en el presente artículo, es la de Extremera, Durán y Rey (2005), quienes analizaron la relación entre los niveles de inteligencia emocional percibida y satisfacción vital, felicidad subjetiva y engagement. La muestra estuvo conformada por personal asistencial que trabaja con personas con discapacidad intelectual en 
un centro de Málaga, España. Evidenciaron que existe relación entre la felicidad subjetiva y los tres componentes de engagement, alcanzado el índice más elevado con vigor. Concluyen que la comprensión y el manejo emocional se relacionan con el bienestar personal y laboral, con un mayor sentido de compromiso y dedicación hacia su labor. Por ende, recomiendan, para las organizaciones, programas de formación en habilidades socio-emocionales.

Con respecto a la felicidad, el autor pionero en América Latina es Alarcón (Caycho, 2010). A partir de sus investigaciones, otros psicólogos inician sus indagaciones en este nuevo campo en la región. En 2001, investigó si había relación entre felicidad, género, edad y estado conyugal en una población diversa en Lima. En el 2002, Alarcón se propuso identificar los objetos preferidos para alcanzar la felicidad y comprobar si estos están relacionados con el género, estado civil y la edad en una población en Lima. Encontró que los objetos preferidos por los encuestados fueron los siguientes: gozar de buena salud, estar bien con Dios y tener una familia estable. En el 2006, Alarcón realizó la elaboración de la Escala Factorial para medir la felicidad. La escala fue aplicada a una población universitaria en Lima. Esta Escala Factorial para medir la Felicidad es la que se emplea en este trabajo.

Se han encontrado estudios para medir la felicidad en poblaciones diversas a nivel nacional (Alarcón, 2001; 2002; Fernández, 2008) internacional (Peiró, 2001; Bilbao, Techio y Páez, 2007; Pavez, Mena y Vera-Villarroel, 2012). Asimismo, se ha aplicado en poblaciones universitarias en el Perú (Alarcón, 2006, 2009; Condori, 2013) y en otros países (Posada y Aguilar, 2012; Torres, Moyano-Díaz y Páez, 2014).

Las investigaciones sobre engagement han sido aplicadas en ámbitos laborales y a nivel internacional. Moreno-Jiménez, Garrosa, Corso, Boada y RodriguezCarvajal (2012) examinaron los efectos de las variables sociodemográficas, personales y personalidad resistente con las variables agotamiento y vigor del engagement en una población mixta (enfermeras y docentes) en España y Perú. Asimismo, Garrosa, Blanco-Donoso, Moreno-Jiménez, González y Fraca (2014) investigaron la relación del sentido de la coherencia y la reevaluación cognitiva con el work engagement en voluntarios en España. Por su parte, Oramas, Marrero, Cepero, Del Castillo y Vergara (2014) analizaron la relación entre el work engagement y las variables sociodemográficas (sexo, edad y nivel de escolaridad) en trabajadores cubanos.

Con respecto a la medición de engagement, esta ha sido abordada de manera independiente en población diversa (Moreno-Jiménez et al., 2012; Garrosa et al., 2014; Oramas et al., 2014;) y en población universitaria a nivel internacional (Manzano, 2002; Casuso, 2011; Parra, 2010; Glaria et al., 2015; López-Alonso, López-Aguado, Fernández-Martínez, Liébana y Gutiérrez-Provecho, 2016). Para 
el contexto universitario peruano, se citan los trabajos de Moreno-Jiménez et al., 2012, y de Lozano y Reyes, 2017.

De esta manera, se observa que todavía no se ha indagado en torno a la correlación de las variables felicidad y engagement en ámbitos universitarios en el Perú. En este sentido, la presente investigación busca constituirse como un aporte para este nuevo enfoque de la psicología positiva aplicada a las organizaciones $y$, más específicamente, al ámbito de las instituciones de estudios superiores en el Perú.

En la muestra de la presente investigación, se tiene estudiantes universitarios y técnicos que trabajan o realizan prácticas pre profesionales, lo cual implica un doble esfuerzo y dedicación para alcanzar un buen desempeño en ambas organizaciones a las que pertenecen (la empresa y la institución académica). Por ello, este estudio tiene pertinencia aplicativa, ya que los resultados permitirán a los gestores del desarrollo organizacional y del talento poder involucrar a las organizaciones para que estas presten mayor atención al compromiso y al vínculo con esta población, realizando actividades tendientes a incrementar el nivel de engagement.

Consecuentemente, las preguntas de investigación que se plantean son las siguientes: 1) ¿existe correlación entre felicidad y engagement en estudiantestrabajadores de educación superior en Lima, Perú?, 2) ¿cuál es el nivel de felicidad y engagement en estudiantes de educación superior que estudian y trabajan?, y 3) ¿existe correlación entre los factores de la felicidad y los componentes de engagement en estudiantes de educación superior que trabajan en Lima?

Las hipótesis son las siguientes: 1) sí existe correlación directa y significativa entre felicidad y engagement en estudiantes-trabajadores de educación superior en Lima, Perú, 2) los estudiantes de educación superior que estudian y trabajan poseen un nivel alto en felicidad y engagement, y 3) sí existe correlación directa y significativa entre los factores de la felicidad y los componentes de engagement en esta población.

\section{MÉTODO}

\section{Población y Muestra}

La población de la presente investigación estuvo conformada por 2800 estudiantes de universidades e institutos del distrito de Santiago de Surco, quienes trabajan y estudian las carreras de letras y números, de ler a 4to año.

La muestra estuvo conformada por 338 estudiantes, con un 95\% de confianza y un $5 \%$ de margen de error, obtenido a través de la siguiente fórmula: 


$$
n=\frac{Z^{2} \sigma^{2} N}{E^{2}(N-1)+Z^{2} \sigma^{2}}
$$

Valores:

n: Tamaño de la muestra

N: Tamaño de la población

Z: Valor de la distribución estandarizada correspondiente al nivel de confianza. Por lo tanto, como el $\mathrm{NC}=95 \%$, entonces $Z=1.96$

E: Máximo error estándar, donde $\mathrm{E}=5 \%=0.05$

$\sigma^{2}: 0.25$

El tipo de muestreo es no probabilístico intencional, ya que los participantes fueron seleccionados tomando en cuenta los siguientes criterios:

Criterios de inclusión:

Edad: 18 a 40 años

Año académico: 1ero a 4to

Condición laboral: Actualmente trabajando

Carreras: Ligadas al área de letras (Administración, Comunicación y Psicología) y números (Ingeniería y Contabilidad).

Se delimitó tres rangos de edad: de 18 a 23 años, de 24 a 30 años, de 31 a 40 años, tomando en cuenta la clasificación que propone Papalia, Wendkos y Duskin (2010). Se consideró la edad inicial de 18 años, puesto que corresponde con el promedio de edad de la mayoría de los ingresantes a las universidades (Universia, 2011). Adicionalmente, esta es la edad legal para iniciar un trabajo remunerado.

\section{Instrumentos y Materiales}

La Escala de Felicidad de Lima de Reynaldo Alarcón (2006) fue la prueba que se empleó en la presente muestra. Consta de 27 preguntas. Es una escala tipo Likert con cinco alternativas. La administración de la Escala es individual o colectiva, la cual dura aproximadamente 20 minutos. Se puede aplicar a adolescentes y adultos con edades comprendidas entre los 20 y 35 años. La calificación es manual, los participantes marcan sus respuestas por escrito. Respecto a la tipificación, posee puntajes naturales y percentiles, en el total de una muestra de población general. Como estructura factorial posee 4 subcomponentes o factores: ausencia 
de sufrimiento Profundo, satisfacción con la vida, realización personal, alegría de vivir.

Estudios psicométricos de la prueba original

Respecto a la validez en relación con otras variables, se correlacionó la Escala de Felicidad y el Inventario de Personalidad de Eysenck, EPI (Anicama, 1985, citado por Alarcón 2006), en una muestra de 298 estudiantes universitarios. Con respecto a la relación con la Escala de Felicidad y extraversión se halló una correlación alta y significativa $(\mathrm{r}=.378 ; \mathrm{p}<.01)$, en tanto que con neuroticismo, arrojó ser negativa $\mathrm{y}$ alta $(\mathrm{r}=-.450 ; \mathrm{p}<.01)$. Estos resultados se corresponden con lo descubierto en otros estudios, que demuestran correlaciones significativas y positivas entre felicidad y extroversión, y correlaciones significativas y negativas entre felicidad y neuroticismo. Asimismo, existe correlación entre Felicidad, y Afectos Positivos y Negativos. Al respecto, Alarcón (2006) diseñó una Escala de Afectos Positivos y Negativos, que luego fue correlacionada con la Escala de Felicidad. La correlación de Pearson entre felicidad y afectos positivos es positiva y significativa $(\mathrm{r}=.48$; $\mathrm{p}<.01)$. Entre felicidad y afectos negativos, fue significativa y negativa $(r=-.51$; $\mathrm{p}<.01$ ). En otras palabras, se evidencia una correlación altamente significativa entre satisfacción con la vida y frecuencia de afectos positivos $(r=.406, p<.01)$. Al correlacionar la Escala de Felicidad con introversión y neuroticismo, y con afectos positivos y negativos, se sustentó la validez del criterio.

Finalmente, para la validez factorial de la escala, se encontró que esta posee 4 áreas bien definidas, lo que permite que el constructo obtenga coherencia entre sus medidas. (Alarcón, 2006, p. 104)

Coeficiente de Confiabilidad:

Se encontró que las correlaciones en su totalidad son altamente significativas $(\mathrm{p}<.001$, para 2 colas) respecto a la correlación Ítem-Escala. La media es también elevada $(\mathrm{r}=.511 ; \mathrm{p}<.001)$. Estos resultados evidencian que los ítems miden un mismo constructo y que colaboran eficazmente con el puntaje total. Respecto a las correlaciones Ítem-Ítem, la media indica una relación significativa entre los ítems de la Escala $(\mathrm{r}=.28 ; \mathrm{p}<.01)$. No obstante, cada reactivo conserva su autonomía, dentro de la Escala como un todo, puesto que demuestran diferencias con los otros. Asimismo, se evidencian diferencias significativas $(\mathrm{F}=212.69, \mathrm{p}<.01)$ a partir del Análisis de Varianzas entre la medias de los reactivos. Respecto al Coeficiente Alfa de Cronbach, Alarcón (2006) señala que todos los valores Alfa son altamente significativos. Su rango va de .909 a .915 . La Escala total presenta una elevada consistencia interna ( $\alpha=.916$, basada en ítems estandarizados). Finalmente, respecto a los coeficientes Split-Half, se ha determinado el coeficiente de mitades de Spearman-Brown (longitudes desiguales) $\mathrm{r}^{\mathrm{n}}=.884$ y el de Guttman Split-Half 
$\mathrm{r}=.882$. Ambas magnitudes de las correlaciones respaldan la alta confiabilidad de la Escala.

Ficha Técnica: Cuestionario Engagement (UWES)

Se empleó el Cuestionario Engagement (Utrecht Work Engagement Scale UWES) de Marisa Salanova, Wilmar B. Shaufeli, Susana Llorens, Jose M. Peiro, Rosa Grau de España (2001), costa de 17 items. La administración del cuestionario es individual o colectiva. La duración de la aplicación es de aproximadamente 20 minutos. Puede ser aplicable a adolescentes y adultos con edades comprendidas entre los 19 y 58 años, siendo estos trabajadores hombres o mujeres de diferentes ocupaciones y sectores socio- económicos. Al participante se le entrega el cuestionario impreso, en el cual marca sus respuestas. Respecto a la puntuación se califica de manera manual. Suele usarse en el ámbito organizacional, especialmente por profesionales que se desempeñan como expertos en prevención de riesgos laborales o del departamento de Recursos Humanos de las empresas. Posee como estructura factorial 3 subcomponentes: Vigor, Dedicación, Absorción.

Estudios psicométricos de la prueba original

El manual preliminar del UWES - Utrecht Work Engagement Scale, elaborado por Schaufeli y Bakker (2003, citado por Valdez y Ron, 2011), fue traducido por Valdez y Ron (2011) a partir del trabajo realizado por Benavides-Pereira, Fraiz de Camargo y Porto (2009, citado por Valdez y Ron 2011), quienes realizaron la primera introducción a la primera versión al español del UWES. Dicho grupo perteneció al Grupo de Estudos e Pesquisas sobre Estresse e Burnout.

Respecto a la validez y confiabilidad, resumieron los resultados de los análisis psicométricos del UWES indicando que respecto a la validez factorial, los análisis factoriales confirmatorios muestran que la estructura hipotética del UWES de tres componentes, es superior al modelo de un solo factor y se ajusta a los datos de las diversas muestras tomadas en Holanda, España y Portugal. Respecto a la estructura interna, el análisis factorial confirmatorio del UWES define una estructura tridimensional. Estos tres componentes están íntimamente relacionados. Las correlaciones entre las tres escalas generalmente exceden el .65. La correlación entre las variables latentes presentan un intervalo entre .80 y .90 . Respecto a la consistencia interna de las tres escalas del UWES es adecuada. En todos los casos, los valores $\alpha$ de Cronbach son iguales o superan el valor crítico de .70. Usualmente, los valores $\alpha$ de Cronbach varían entre .80 y .90 (cita a Salanova et al., 2000). Respecto a la estabilidad, los puntajes del UWES son relativamente estables a través del tiempo y presentan una estabilidad promedio de dos años para los coeficientes de vigor, dedicación y absorción, los cuales son .30, .36 y .46 respectivamente. 


\section{Procedimiento}

La información fue obtenida con la aceptación de los estudiantes para completar el cuestionario y la escala en esta investigación. Se solicitó a todos los participantes contestar todos los reactivos recibidos en las hojas impresas, considerando las mismas instrucciones de cada prueba. Se aplicó de manera individual y/o colectiva en grupos de no más de 30 participantes por vez.

La autora de la investigación se encargó de la aplicación de las pruebas y también tuvo el apoyo de dos docentes universitarios quienes aplicaron las pruebas en secciones a su cargo. Se retiraron las pruebas que fueron respondidas de manera incompleta.

\section{Análisis de datos y resultados}

\section{Prueba de normalidad de Felicidad y Engagement}

La presente Tabla 1 (pág. 397) muestra que los resultados (salvo Felicidad) tienen una distribución no normal en ambas variables $(\mathrm{p}<0.05)$; por lo tanto, se justifica el uso de estadística no paramétrica.

\section{Análisis de Correlación entre Felicidad y Engagement}

En la Tabla 2 se aprecia que entre Felicidad y Engagement existe una correlación estadísticamente muy significativa, de tipo directo y de nivel moderado bajo, $\mathrm{r}=$ $.349, \mathrm{p}<.001$, con un tamaño del efecto de 0.595 , lo que implica que el engagement explica en un $59.5 \%$ a la felicidad, es decir, presenta una intensidad moderada.

\section{Tabla 2}

Correlación entre Felicidad y Engagement

\begin{tabular}{llc}
\hline & & Engagement \\
\hline \multirow{3}{*}{ Felicidad } & Rho de Spearman & $.349^{* *}$ \\
& rs2 & 0.595 \\
& Sig. (bilateral) & .000 \\
\hline
\end{tabular}

$* * \mathrm{p}<0.01$ Muy significativo

$* \mathrm{p}<0.05$ Significativo

\section{Identificación de niveles de Felicidad y Engagement}

En la Tabla 3 se observa los resultados de frecuencias y porcentajes, así como los resultados inferenciales del análisis prueba de bondad de ajuste a un modelo de niveles iguales, encontrando niveles diferentes de manera estadísticamente significativa, tanto en Felicidad $\left(\chi^{2}=80.5, \mathrm{p}<.001\right)$ como en Engagement $\left(\chi^{2}=\right.$ $131.0, \mathrm{p}<.001)$. Con respecto al análisis de niveles de ambas variables, se encontró 





que el 60\% (203) de estudiantes se ubican en el nivel Alto en Engagement, con la particularidad que no se encontró ningún estudiante en los niveles bajo y muy bajo. En cambio, el 38\% (127) de los estudiantes alcanzaron un nivel medio en Felicidad.

\section{Tabla 3}

Niveles de Felicidad y Engagement

\begin{tabular}{lccccccccccccc}
\hline Variables & \multicolumn{1}{c}{ Muy bajo } & \multicolumn{2}{c}{ Bajo } & \multicolumn{2}{c}{ Medio } & \multicolumn{2}{c}{ Alto } & Muy alto & $\chi^{2}$ & $\boldsymbol{p}$ & $\mathbf{V}$ \\
\hline Felicidad & 41 & $12 \%$ & 41 & $12 \%$ & 127 & $38 \%$ & 80 & $24 \%$ & 49 & $14 \%$ & 80.5 & $<.001$ & 0.49 \\
Engagement & 0 & $0 \%$ & 0 & $0 \%$ & 103 & $30 \%$ & 203 & $60 \%$ & 32 & $9 \%$ & 131.0 & $<.001$ & 0.62 \\
\hline
\end{tabular}

Nota. El porcentaje total no es 100 en Engagement por aspectos del redondeo.

\section{Análisis de Correlación entre los factores de Felicidad y los componentes de Engagement}

En la Tabla 4 se observa que el factor Ausencia de sufrimiento profundo (perteneciente a felicidad) correlaciona de manera directa y muy significativamente con los componentes de Engagement, teniéndose las mayores puntuaciones en Dedicación, $r_{s}=.386, p<.001$, Vigor $r_{s}=.345, p<.001$, así como con el total, $r_{s}=.311, p<.001$. En Satisfacción con la vida (factor de la felicidad), la correlación más alta se tiene con Dedicación, $r_{s}=346$, luego con vigor, $r_{s}=.307, \mathrm{p}$ $<.001$, mientras que con Engagement se tiene $r_{s}=288, p<.001$. Para el factor de Realización personal (perteneciente a felicidad), se tiene la mayor correlación con Dedicación, $r_{s}=.371, p<.001$, luego con Vigor, $r_{s}=.330, p<.001$, mientras que con Engagement se tiene $r_{s}=.304, p<.001$. Con respecto a Alegría de vivir (factor de la felicidad), el mayor grado de correlación se da con Dedicación, $r_{s}=.329, p$ $<.001$, mientras que con Engagement la significación es pequeña $r_{s}=.277, p<$ .001. Finalmente, con respecto a Felicidad total, la mayor correlación se tiene con Dedicación, $r_{s}=.430, p<.001$, y luego con Vigor, $r_{s}=.382, p<.001$.

Tabla 4

Correlación entre los factores de Felicidad y los componentes de Engagement

\begin{tabular}{llccc}
\hline & & Eng. Vigor & Eng. Dedicación & Eng. Absorción \\
\hline Fel. Ausencia de & Rho de Spearman & $.345^{* *}$ & $.386^{* * *}$ & $.144^{* *}$ \\
sufrimiento profundo & Sig. (bilateral) & .000 & .000 & .007 \\
Fel. Satisfacción con & Rho de Spearman & $.307^{* *}$ & $.346^{* *}$ & $.144^{* * *}$ \\
la vida & Sig. (bilateral) & .000 & .000 & .007 \\
Fel. Realización & Rho de Spearman & $.330^{* *}$ & $.371^{* *}$ & $.151^{* *}$ \\
personal & Sig. (bilateral) & .000 & .000 & .005 \\
Fel. Alegría de vivir & Rho de Spearman & $.303^{* *}$ & $.329^{* *}$ & $.132^{*}$ \\
& Sig. (bilateral) & .000 & .000 & .014 \\
\hline
\end{tabular}

$* * \mathrm{p}<0.01$ Muy significativo

$* \mathrm{p}<0.05$ Significativo 
Por ende, se concluye que existe relación directa y muy significativa entre los factores de la Felicidad y los componentes del Engagement en estudiantes de educación superior que trabajan en Lima.

\section{Discusión de los Resultados}

\section{Análisis de los resultados referidos a la relación entre Felicidady Engagement}

Los resultados muestran que existe relación positiva, muy significativa, de un nivel moderado bajo entre los puntajes totales de felicidad y engagement en la población analizada, es decir, a mayor felicidad mayor engagement, afectándose mutuamente en un 59\%. Resultados similares fueron obtenidos por Extremera et al. (2005) quienes, en una muestra de personal asistencial de España, hallaron relaciones positivas entre la felicidad subjetiva y los tres componentes de engagement. Al respecto, Salanova y Schaufeli (2009) mencionan que las personas eligen tareas para las que se sienten capaces y motivados, contando con un clima positivo de equipo, de compañeros de aula y familia, sintiéndose orgullosos de sí mismos, felices y viendo su situación laboral y académica como un reto. Este estado de felicidad hace incrementar el engagement de los involucrados como si fuera un proceso de contagio emocional. En esta misma línea, Lyubomirsky (2011) considera a la combinación de esfuerzo y dedicación como un paso para alcanzar la Felicidad.

\section{Análisis de la identificación de niveles de Felicidad y Engagement}

Se encontró que el 38\% de participantes se ubican en un nivel medio de Felicidad, con una tendencia al nivel alto. Al respecto hay concordancia con los reportes de Cuadra y Florenzano (2003), quienes hallaron que los estudiantes que se encuentran comprometidos en actividades interesantes (ya sea su carrera o su trabajo) tienden a experimentar mayor satisfacción con su vida. La actividad en sí misma se convierte en un motivador que incluso supera la retribución material (Seligman, 2006), ya que sienten que dichas actividades les permiten sentir que contribuyen con un bien mayor como la realización personal. En esta línea, Haidt señala que "la felicidad procede del interior, pero también del exterior" (2006, p. 132), es decir, que los estudiantes encuentran un sentido a su vida, cuando encuentran oportunidades para trascender, dejando un legado a los demás.

A nivel del engagement, el $60 \%$ de la muestra se ubica en un nivel Alto, observándose que no existen estudiantes con niveles bajo y muy bajo. Salanova y Schaufeli explican que las personas con alto engagement manifiestan una "conexión enérgica y efectiva con sus trabajos, y se ven capaces de afrontar las nuevas demandas que aparecen en el día a día laboral" (2004, p. 115). Así mismo, para que el engagement se presente, es importante que la empresa fomente el feedback constructivo, logre un clima laboral positivo, dando oportunidad 
a que los trabajadores experimenten una variedad de tareas con la supervisión adecuada (a tiempo, sin autoritarismo), orientada a brindar oportunidades para el aprendizaje y el desarrollo personal. Se infiere, por lo tanto, que los participantes poseen estrategias para ver las actividades laborales que realizan con optimismo, se perciben autoeficaces, es decir, con capacidad de reducir o amortiguar el potencial impacto negativo de las demandas laborales y con un adecuado nivel de autoestima, como evidencian Salanova y Schaufeli (2009). El presente resultado es favorable para el estudiante que trabaja, ya que al no presentar o tener menos síntomas físicos y psicológicos por estrés, puede tener dos actividades demandantes, trabajar y estudiar (Casuso, 2011).

\section{Análisis de correlación entre los factores de Felicidad y los componentes de Engagement}

Al analizar la correlación entre los factores de felicidad y los componentes de engagement, se encontró lo siguiente:

Existe correlación estadísticamente significativa, $r_{s}(336)=.35, p<.001$, entre los factores de felicidad y los componentes de engagement. Con ello, se demuestra la afirmación de Haidt (2006) encontrando que uno de los componentes de la fórmula para conocer el nivel de felicidad que experimentan las personas está determinado por las actividades voluntarias que realizan. En la presente investigación, las actividades voluntarias serían las actividades laborales que son realizadas con vigor, dedicación, absorción (componentes de engagement). Tomando lo señalado por Garrosa et al. (2014), los estudiantes que le dan un significado a lo que hacen y perciben dominio y confianza en sus recursos personales, se sienten capaces de enfrentar las demandas que se le presenten y evidencian más bienestar y mayor efecto positivo.

El componente dedicación de la variable engagement se correlaciona con todas los factores de la variable felicidad. Ello puede deberse a que el componente mencionado es un componente emocional del engagement, referida a una alta implicación laboral, junto con la manifestación de un sentimiento de significación, entusiasmo, inspiración, orgullo y reto por el trabajo.

Así mismo, el componente Vigor de la variable engagement se correlaciona con todos los componentes de la variable felicidad. El componente vigor es un componente conductual - enérgico de la variable Engagement, que implica altos niveles de energía y resistencia mental mientras se trabaja, debido al deseo de esforzarse en el trabajo incluso cuando se presentan dificultades. Implica tener determinación y fortaleza, por ello, se entiende que se correlacione con el factor ausencia de sufrimiento profundo, llamado también por Alarcón como sentido positivo de la vida. Al respecto, los autores Extremera, Durán y Rey (2005) 
también encontraron relación entre felicidad subjetiva y los tres componentes de engagement, siendo vigor el componente que alcanzó el índice más elevado.

El componente Absorción de engagement, que es un componente cognitivo del engagement, se correlaciona de manera muy significativa con ausencia de sufrimiento profundo, satisfacción con la vida, realización personal y felicidad total y de manera significativa con alegría de vivir. El componente absorción se refiere a que la persona está totalmente concentrada en su trabajo, debido a las fuertes dosis de disfrute y concentración experimentadas.

\section{Conclusiones}

1. Existe relación directa y muy significativa entre Felicidad y Engagement en estudiantes de nivel superior que están trabajando en Lima. Siendo el tamaño del efecto de 0.59 , considerado como moderado.

2. El $60 \%$ de estudiantes alcanzaron un nivel alto de Engagement, mientras que el $38 \%$ se ubica en un nivel medio en Felicidad.

3. La Dedicación y el Vigor del Engagement, al ser componentes conductualenérgico y componente emocional se correlacionan con los factores de la Felicidad que implican emoción y acción. El componente Absorción de Engagement, que es un componente cognitivo del engagement, se correlaciona de manera muy significativa con Ausencia de Sufrimiento profundo, Satisfacción con la vida, Realización Personal y Felicidad total y de manera significativa con Alegría de vivir.

\section{REFERENCIAS}

Alarcón, R. (2009). Psicología de la Felicidad, introducción a la psicología positiva. Lima: Universidad Ricardo Palma.

Alarcón, R. (2006). Desarrollo de una Escala Factorial para Medir la Felicidad. Revista Interamericana de Psicología, 40(1) pp. 99-106. Recuperado de https://goo.gl/ sHRHGi

Alarcón, R. (2002). Fuentes de felicidad: ¿Qué hace feliz a la gente? Revista de Psicología de la PUCP, XX(2), 170-196. Recuperado de https://goo.gl/R5FqzB

Alarcón, R. (2001). Relaciones entre felicidad, género, edad y estado conyugal. Lima: Revista de Psicología de la PUCP, XIX(1), 28-46.

Bilbao, M., Techio, E., y Páez, D. (2007). Felicidad, cultura y valores personales: estado de la cuestión y síntesis meta-analítica. Lima: Fondo Editorial PUCP Revista de Psicología, XXV(2). Recuperado de https://goo.gl/R9DzpA

Carr, A. (2007). Psicología Positiva: La Ciencia de la Felicidad. Barcelona: Paidós. Recuperado de https://goo.gl/48Je58 
Casuso, M. J. (2011). Estudio del estrés, engagement y rendimiento académico en estudiantes universitarios de ciencias de la salud. Málaga: SPICUM Servicio de publicaciones. Recuperado de https://goo.gl/A54qec

Caycho, T. (2010). Reseña de la obra Psicología de la felicidad. Introducción a la psicología positiva de Alarcón, R. (2009). Recuperada de https://goo.gl/ciWfaF

Condori, L. (2013). Relación de: Autoeficacia ante el estrés, personalidad, percepción del bienestar psicológico y de salud con el rendimiento académico en universitarios. Lima: Tesis para optar el Grado académico de Doctor en Psicología UNMSM. Recuperada de https://goo.gl/4UhvPr

Costa, A. (24 de abril de 2015). Perú es uno de los cuatro países menos felices de Latinoamérica). El Comercio. Recuperado de https://goo.gl/TNEu8p

Csikszentmihalyi, M. (2008). Fluir (Flow): una psicología de la felicidad. Barcelona: Kairós: Debolsillo.

Cuadra, H., y Florenzano, U. (2003). El Bienestar Subjetivo: Hacia una Psicología Positiva. Chile: Revista de Psicología de la Universidad de Chile, XII(1), 83-96. Recuperado de https://goo.gl/MWSASw

Extremera, N., Durán, A., y Rey, L. (2005). La inteligencia emocional percibida y su influencia sobre la satisfacción vital, la felicidad subjetiva y el engagement en trabajadores de centros para personas con discapacidad intelectual. España: Ansiedad y Estrés. Recuperado de https://goo.gl/brKh4w

Fernández, M. (2008). Burnout, autoeficacia y el estrés en maestros peruanos: tres estudios facticos. Ciencia \& Trabajo, 30, 120-125. Recuperado de https://goo.gl/8j6enb

Garrosa, E., Blanco-Donoso, L., Moreno-Jiménez, B., González, A., y Fraca, M. (2014). Evaluación y predicción del work engagement en voluntarios: El papel del sentido de la coherencia y la reevaluación cognitiva. Anales De Psicología, 30(2), 530-540. doi:http://dx.doi.org/10.6018/analesps.30.2.148701

Glaría, R., Carmona, L., Pérez, C., y Parra, P. (2015). Burnout y engagement académico en fonoaudiología. Investigación en Educación Médica. Recuperado de https://goo. $\mathrm{gl} / \mathrm{r} 3 \mathrm{UP} 48$

Haidt, J. (2006). La hipótesis de la felicidad. España: Gedisa Editorial.

León, J., y Sugimaro, C. (2013) Entre el estudio y el trabajo: Las decisiones de los jóvenes peruanos después de concluir la educación básica regular. Avances de Investigación 11. Lima: Grupo de Análisis para el Desarrollo, GRADE. Recuperado de https://goo. $\mathrm{gl} / \mathrm{rEsMDz}$

López-Alonso, A., López-Aguado, M., Fernández-Martínez, M., Liébana, C., y GutiérrezProvecho, L. (2016). Los enfoques de aprendizajes, en engagement, el ocio y el rendimiento anterior, propuesta de un modelo. Revista de Pedagogía, 68(2). doi: http://dx.doi.org/10.13042/Bordon.2016.40940

Lozano-Paz, C. y Reyes-Bossio, M. (2017). Docentes universitarios: Una mirada desde la autoeficacia general y engagement laboral. Revista Digital de Investigación en Docencia Universitaria, 11(1), 134-148. doi: http://dx.doi.org/10.19083/ridu.11.503 
Lyubomirsky, S. (2011) La ciencia de la felicidad. Barcelona: Ediciones Urano.

Manzano, G. (2002). Burnout y Engagement en un colectivo preprofesional, estudiantes universitarios. Boletín de Psicología, 74, 79-102. Revisado de https://goo.gl/ERqtjG

Moreno-Jiménez, B., Garrosa, E., Corso, S., Boada, M., y Rodríguez-Carvajal, R. (2012). Personalidad resistente y capital psicológico: las variables personales positivas y los procesos de agotamiento y vigor. España: Psicothema, 24(1), 79-86.

Oramas, A., Marrero, I., Cepero, E., Del Castillo, N., y Vergara, A. (2014). La escala de 'work engagement' de Utrech. Evaluación del 'work engagement' en trabajadores cubanos. Revista Cubana de Salud y Trabajo, 15(2), 47-56. Recuperado de https:// goo.gl/oaDRM6

Organización Internacional del Trabajo -OIT (2011). Panorama Laboral 2011 América Latina y el Caribe. Lima: OIT /Oficina Regional para América Latina y el Caribe. Recuperado de https://goo.gl/QMRoCS

Organización Internacional del Trabajo -OIT (2015). Panorama Laboral 2015 América Latina y el Caribe. Lima: OIT /Oficina Regional para América Latina y el Caribe. Recuperado de https://goo.gl/zzxvV3

Padrós, F. (2002). Disfrute y Bienestar Subjetivo. Un estudio psicométrico de la Gaudibilidad. Recuperado de https://goo.gl/yGd1Sq

Papalia, D., Wendkos, S. y Duskin, R. (2010) Desarrollo Humano. México: McGraw-Hill Educación.

Parra, P. (2010). Relación entre el nivel de Engagement y el rendimiento académico teórico/ práctico. Rev Educ Cienc Salud, 7(1), 57-63. Recuperado de https://goo.gl/MF6zbm

Pavez, P., Mena, L. y Vera-Villarroel, P. (2012). El rol de la felicidad y el optimismo como factor protector de la ansiedad. Universitas Psychologica, 11 (2), 369-380. Recuperado de https://goo.gl/R1sqXQ

Peiró, A. (2001). Condiciones socioeconómicas y felicidad en los españoles. España: Instituto Valenciano de Investigaciones económicas S.A.

Posada, R., y Aguilar, O. (2012). La felicidad como elemento del desarrollo sostenible desde la organización. Revista del Centro de Investigación. Universidad La Salle, 10(37), 83-94. Obtenido el 15 de junio de 2013, de https://goo.gl/zSKsVs

Quintanilla, I. (2013). Psicología social del trabajo. Madrid: Ediciones Pirámide.

Salanova, M., Grau, R., Llorens, S. y Schaufeli, W. (2001). Exposición a las tecnologías de la información, burnout y engagement: el rol modulador de la autoeficacia profesional. Revista de Psicología Social Aplicada, 11(1), 69-90. Recuperado de https://goo.gl/rq1YLi

Salanova, M., y Schaufeli, W. (2009) El engagement en el trabajo, cuando el trabajo se convierte en pasión. España: Alianza Editorial.

Salanova, M., y Schaufeli, W. (2004). El engagement de los empleados: Un reto emergente para la Dirección de los Recursos Humanos. Estudios Financieros, 261(62), 109-138. Recuperado de https://goo.gl/g1hWY6 
Salanova, M., Schaufeli, W., Llorens, S., Peiro J.M. y Grau, R. (2000) Desde el "burnout" al "engagement": ¿una nueva perspectiva? Revista de Psicología del Trabajo y de las Organizaciones, 16(2), 117-134. Recuperado de https://goo.gl/XjTcXy

Salas, J. (2010). Bienestar Psicológico y síndrome de Burnout en docentes de la UGEL $N^{\circ}$ 7 (Tesis de maestría). Recuperada de https://goo.gl/azK7YC

Schaufeli, W., Salanova, M., González-Romá, V. y Bakker, A. (2001) The measurement of engagement and burnout: A two sample confirmatory factor analytic approach. Journal of Happiness Studies, 3, 71-92. Recuperado de https://goo.gl/2bjdy5

Seligman, M. (2006). La auténtica Felicidad. Barcelona: Vergara grupo Z.

Torres, M., Moyano-Díaz, E., y Páez, D. (2014). Comportamiento juvenil universitario en busca de la felicidad: su caracterización y su eficacia. Universitas Psychologica, 13(4), 1419-1428. doi: http://dx.doi.org/10.11144/Javeriana.UPSY13-4.cjub

Valdez, H. y Ron, C. (2011). UWES Utrecht Work Engagement Scale: Escala Utrecht de Engagement en el Trabajo. Recuperado de https://goo.gl/zAW6jm 